readability to how they appeal to patients. Previous studies have demonstrated a positive impact of visual media across the range of patients' literacy skills, both health-specific and generic.

Objectives To devise a production mechanism for serious games in patient education aligned with the current development of patient guidelines, keeping costs manageable in the long term.

Methods The MUSE FP7 Project, funded by the European Commission, brings together computer scientists, cognitive psychologists and one GIN member. The project investigates the long-term potential of automatic generation of serious games from patient guidelines documents. It uses state-of-the art commercial gaming technology as well as developing new text analysis software.

Results A first prototype has been developed on the topic of bariatric surgery education. It features male and female patient avatars in a hospital environment and supports the interactive exploration and rehearsal of the various stages of the process. All the game actions can be related to specific portions of the patient education document.

Discussion The popularity of new media such as computer games improves dissemination prospects for patient education information. In addition, as suggested by recent research, the interactive nature of serious games makes the information more accessible, facilitates learning and addresses issues not covered by textual dissemination such as patient anxiety.

Implications for Guideline Developers/Users Serious gaming is poised to become a major health-related medium, hence the need for specific development processes.

\section{P311 A SURVEY ON THE LIKELY RESOURCES OF GUIDELINE DISSEMINATION AND THE PERCEIVED BARRIERS TO THE UTILIZATION IN KOREA}

${ }^{1} \mathrm{E}$ Shin, ${ }^{2} \mathrm{H}$ Jo, ${ }^{3} \mathrm{~S}$ Lee, ${ }^{4} \mathrm{~S}$ Chang, ${ }^{5} \mathrm{M}$ Oh. ${ }^{1}$ Department of Preventive Medicine \& Public Health, Ajou University School of Medicine, Suwon, South Korea; ${ }^{2}$ Department of Health Management and Policy, Kangwon National University School of Medicine, Chuncheon, South Korea; ${ }^{3}$ Department of Preventive Medicine, Ewha Womans University School of Medicine, Seoul, South Korea; ${ }^{4}$ Department of Urology, Kyung Hee University School of Medicine, Seoul, South Korea; ${ }^{5}$ Department of Health Management and Policy, Kangwon National University School of Medicine, Chuncheon, South Korea

\section{0:1136/bmjqs-2013-002293.251}

Background Dissemination resources are very important to utilise Guidelines. To provide useful information to make a strategy for promoting implementation effectiveness, identifying the barriers to the utilisation should be done.

Objectives To identify the likely resources of guideline dissemination and find out the barriers to the utilisation of a clinical practice guideline in Korea.

Methods Likely resources of dissemination and the perceived barriers to the utilisation of a clinical practice guideline for sexually transmitted infections which is developed in 2011 by The Korean Urological Association were surveyed using structured questionnaire by e-mailing to physicians working at primary health care clinics from 1st Nov - 30thNov 2012. Total number of respondents was 305 and the response rate was $6 \%$.

Results There were various likely resources of a guideline dissemination; the most likely resource was a printed full version guideline (76.3\%), followed by web and mobile application (Guideline APP) (65.6\%) and learning modules such as workshop (51.5\%). Barriers to the utilisation of clinical practice guideline were 'not knowing because of the improper promotion', 'unclear compensatory mechanisms like the fee schedule for practice guidelines', and 'insufficient treatment time to check the recommendation of practice guidelines'.

Discussion To maximise guidelines dissemination benefits, various resources should be provided at the same time. The current surveyed knowledge of barriers may be very important information for implementing guidelines.

Implications for Guideline Developers/Users Identifying resources of dissemination and understanding barriers to the utilisation is important for development efficient tailor-made implementation strategies.

\section{P312 A FRENCH PROJECT OF INTERREGIONAL AND SHARED GUIDELINES IN SUPPORTIVE CARE}

1,2,5 F Farsi, ${ }^{1,3}$ | Klein, ${ }^{1,4} \mathrm{~N}$ Jovenin, ${ }^{1} \mathrm{H}$ Labrosse, ${ }^{1} \mathrm{M}$ Brunet, ${ }^{3} \mathrm{~V}$ Block, ${ }^{1,3,5}$ Krakowski. ${ }^{1}$ AFSOS -French speaking association of supportive care, Paris, France; ${ }^{2}$ Rhône-Alpes Regional Network, Lyon, France; ${ }^{3}$ Lorraine Regional Network, Nancy, France; ${ }^{4}$ Champagne-Ardennes Regional Network, Reims, France; ${ }^{5}$ French Federation of Cancer Centers, Paris, France

\section{0:1136/bmjqs-2013-002293.252}

Background In France, access to supportive care is a right for cancer patients It is enshrined in the health regulations (1st and 2nd national cancer plans). But equity in this access is still evolving. In this context a national learned society and the regional oncology networks are desired to support professionals by organising the sharing and exchanges on the decision support tools

Objectives Gradually enlist all French regional cancer networks and all French experts in selecting the priority thematic, methodological support in the working groups and in objectives of implementation involve the maximum of professionals in the rereading and validation of shared guidelines

Method Establishment of a national committee dedicated to supporting methodological and organisational project. - Needs analysis and choice of themes by regional cancer networks recruitment of experts by both the networks and the learned societies - establishment of inter-disciplinary working group organisation of a re-reading solicitant learned society and cancer networks - National Day (J2R) with dedicated workshops and plenary sessions to confirm or update the guidelines.

Results and Conclusion After 4 years, this project with regional networks complete 30 different shared guidelines and the attended by professionals of all the French regions (oncologist; surgeons, supportive care specialists, nurses).

\section{P314 BARRIERS AND FACILITATORS TO THE IMPLEMENTATION OF CLINICAL PRACTICE GUIDELINES: A SURVEY AMONG PHYSICIANS IN SPECIALIZED CARE}

${ }^{1} \mathrm{E}$ Reviriego, ${ }^{2} \mathrm{M}$ Iruretagoyena, ${ }^{3} \mathrm{~A}$ Arcelay, ${ }^{4} \mathrm{G}$ Villanueva. ${ }^{1}$ Osteba - Health Technology Assessment. Health Department. Basque Government, Bilbao, Spain; ${ }^{2}$ Public Health Programs and Patient Safety. Healthcare Management, Osakidetza, Vitoria, Spain; ${ }^{3}$ Healthcare Management. Osakidetza. Basque Health Service, Vitoria, Spain; ${ }^{4}$ Basque Office For Health Technology Assessment, Bilbao, Spain

\section{0:1136/bmjqs-2013-002293.253}

Background In an era when an increasing amount of clinical information is available to health care professionals, the effective implementation of clinical practice guidelines requires the development of strategies to facilitate the use of these guidelines.

Objectives Explore the knowledge and attitudes of Specialised Care Physicians (SC) in terms of the use of CPGs; identify the 
barriers and facilitators to their proper dissemination and implementation; identify the strategies and actions for improvement that contribute to minimising the impact of the barriers that have been detected.

Methods We conducted a survey in order to assess resources, knowledge and attitudinal barriers of physicians working in Specialised Care towards the Clinical Practice Guidelines.

Results The questionnaire was completed by 209 SC physicians. The application of the recommendations in the CPGs is considered to be easy by $61.2 \%$ of participants, while $28.2 \%$ considered this procedure to be difficult. Among the reasons behind the difficulty were: the complicated nature of practical application, the lack of organisational, financial and infrastructurerelated resources, the variable nature of the patients, the lack of time, little evidence with low-quality recommendations, disagreement, a lack of interest and motivation and the lack of knowledge of the CPGs due to unsatisfactory dissemination.

Conclusion Informed by the results of the survey, leading health authorities are making an effort to develop specially designed interventions to implement clinical practice guidelines, including an easily accessible online database.

\section{P317 IMPLEMENTATION OF EVIDENCE BASED HEALTHCARE AND GUIDELINES IN CLINICAL PRACTICE}

${ }^{1} \mathrm{~K}$ Steinhausen, ${ }^{2} \mathrm{~S}$ Slordahl. ${ }^{1}$ Furtwangen University and European Science Foundation, Strasbourg, France; ${ }^{2}$ Norwegian University of Science and Technology and European Science Foundation, Trondheim, Norway

\section{0:1136/bmjqs-2013-002293.254}

Background Healthcare received by Europe's citizens should be based on the best scientific evidence and with involvement of patient and public. Greater emphasis on scientific evidence for a health intervention must be thoroughly analysed, health technology assessment (HTA) must become a cornerstone of healthcare. We have discussed these issues with different interdisciplinary groups and published two strategic papers in 2011 and 2012.

Objectives The aim is to present and discuss further possible implementation steps for improving implementation of evidence based healthcare and guidelines in clinical practice.

Methods In 2011 and 2012 workshops with interdisciplinary working groups (knowledge transfer, patient involvement and general practice) took place. Needs for actions and the relevant stakeholders were identified.

Results Needs for action: Establish a European Institute for Health Research where common issues in European healthcare research and policy can be debated and appropriate strategies formulated. Organise meetings between HTA/EBM leaders and policy-makers and health administrators on the European, national, regional and local level Establish at national level Healthcare Knowledge Centres for improved access to and transfer of unbiased information on patient-oriented research Set up research networks and ensure collaborative research between primary and secondary care Develop incentive systems for using and implementing evidence-based practice, guidelines and policy at medical care level through national European guidelines or even regulations and the relevant stakeholders.

Discussion It is now important to implement these needs. The involvement of different stakeholders from research, clinical practice, regulation, policy, patients and the public is urgently needed.

\section{P319 MINDS PROJECT AS GUIDELINE CLEARINGHOUSE - EVALUATION OF CLINICAL PRACTICE GUIDELINES DEVELOPED IN JAPAN}

${ }^{1,2} \mathrm{~A}$ Okumura, ${ }^{1,3} \mathrm{M}$ Yoshida, ${ }^{1,4} \mathrm{~K}$ Kiyohara, ${ }^{1} \mathrm{~N}$ Takahashi, ${ }^{1,5} \mathrm{Y}$ Hatakeyama, ${ }^{1,6} \mathrm{~N}$ Htun, ${ }^{1,4} \mathrm{Y}$ Sato, ${ }^{1,4} \mathrm{~N}$ Kojimahara, MINDS Group 1, 1,4 N Yamaguch. ${ }^{1} \mathrm{MINDS}$ Center, Japan Council for Quality Health Care, Tokyo, Japan; ${ }^{2}$ Department of Social Medicine, The University of Tokyo, Tokyo, Japan; ${ }^{3}$ Department of Hemodialysis and Surgery, Chemotherapy Research Institute Inter, Tokyo, Japan; ${ }^{4}$ Japan, Department of Public Health , Tokyo, Japan; ${ }^{5}$ Women's Medical University, Tokyo, Department of Advanced Social and International Studies, Graduate School of A, Tokyo, Japan; ${ }^{6}$ Department of Molecular Epidemiology, Tokyo Medical and Dental University, Tokyo, Japan

\section{0:1136/bmjqs-2013-002293.255}

Background MINDS (Medical Information Network Distribution Service) is a consignment project for MHLW (Ministry of Health, Labour and Welfare) managed by Japan Council for Quality Health Care. MINDS has been disseminating evidencebased clinical practice guidelines (CPG) as guideline clearinghouse in Japan.

Objectives To assess the quality of evidence-based CPG developed in Japan.

Methods We searched Japanese CPG using 10 major databases from January 2007 to January 2013. After two-stage screening process with exclusion criteria, identified CPG were evaluated by 4 reviewers of the CPG evaluation group using the AGREEII (Appraisal of Guidelines for Research \& Evaluation II) Instrument.

Results A total of 1763 literatures were identified by the searching process. After screening process, 168 guidelines were evaluated by the AGREEII instrument from September 2011 to January 2013. The scores mean (SD) of each AGREEII domain were as follows: Scope and Purpose, 64.1 (19.2); Stakeholder Involvement, 46.0 (18.2); Rigour of Development, 39.8 (24.6); Clarity of Presentation, 58.8 (21.3); Applicability, 42.7 (16.3); Editorial Independence, 29.9 (31.4) and Overall assessment, 50.4 (21.1).

Discussion Among the AGREEII domains, Editorial Independence and Rigour of Development are important factors to improve the quality of Japanese CPG.

Implications for guideline developers/users It is necessary to cooperate with guideline development group in order to utilise the guidelines evaluation result for improving the guideline development process. MINDS is preparing to hold workshops 2013 focused on guideline methodology for guideline developers.

\section{P321 DISSEMINATION OF THE CLINICAL PRACTICE GUIDELINES DEVELOPMENT METHODOLOGY BASED ON BODY OF EVIDENCE IN JAPAN DEVELOPMENT OF EDUCATIONAL PACKAGE FOR CLINICAL PRACTICE GUIDELINES AND WORKSHOP PROGRAM}

${ }^{1,2} \mathrm{M}$ Yoshida, ${ }^{1,3} \mathrm{Y}$ Hatakeyama , ${ }^{1,4} \mathrm{~A}$ Okumura, ${ }^{1} \mathrm{~N}$ Takahashi, ${ }^{1,5} \mathrm{~N}$ Kojimahara, ${ }^{1,5} \mathrm{~K}$ Kiyohara, ${ }^{1,5} \mathrm{Y}$ Sato, ${ }^{1,6} \mathrm{~N}$ Htun, ${ }^{1,5} \mathrm{~N}$ Yamaguchi. ${ }^{1} \mathrm{MINDS}$ (Medical Information Network Distribution Service) Center, EBM Guidelines, Tokyo, Japan; ${ }^{2}$ Department of Hemodialysis and Surgery, Chemotherapy Research Institute, Inter, Ichikawa, Japan; ${ }^{3}$ Department of Advanced Social and International Studies, Graduate School of A, Tokyo, Japan; ${ }^{4}$ Department of Social Medicine, The University of Tokyo, Tokyo, Japan; ${ }^{5}$ Department of Public Health Tokyo Women Medical University, Tokyo Japan; ${ }^{6}$ Department of Molecular Epidemiology, Tokyo Medical and Dental University, Tokyo, Japan

10:1136/bmjqs-2013-002293.256 